\title{
lleo-uterine fistula in a degenerated posterior wall fibroid after Caesarean section
}

\author{
Ayman Shehata ${ }^{1}$, Naglaa Hussein ${ }^{1}$, Ahmed El Halwagy ${ }^{1}$, Adel El Gergawy' ${ }^{1}$, Mohamed Khairallah ${ }^{2}$ \\ 'Lecturer of Obstetrics and Gynecology, ${ }^{2}$ Lecturer of Surgery, Tanta University, Tanta, Egypt
}

Uterine fibroids are benign tumors of the myometrium with a diverse range of manifestations. Fibroids can dramatically increase in size during pregnancy due to the increase in estrogen levels. After delivery, the fibroids usually shrink back to their pre-pregnancy size. Uterine myomas may have many complications, including abnormal uterine bleeding, infertility, pressure on nearby organs, degeneration, and malignant transformation. No previous reports have indicated that a fistula may develop between a uterine fibroid and the bowel loops, although previous studies have documented the occurrence of fistulas from the uterus to the bowel following myomectomy or uterine artery embolization performed to treat a myoma. In our case report, we document the rare complication of a fistula occurring between a degenerated myoma in the posterior wall and the ileum 1 week postoperatively in a patient who underwent a Caesarean section but did not have a history of uterine artery embolization.

Keywords: Degenerated myoma; Fistula; Uterine fibroids

\section{Introduction}

Uterine myomas, also referred to as fibroids, are benign tumors of the smooth muscle of the uterus and are the most commonly encountered type of tumor in women, occurring in nearly $50 \%-70 \%$ of women of childbearing age [1].

The status of fibroids during pregnancy is especially complex, because fibroids can affect the pregnancy and pregnancy can affect the fibroids. Leiomyomas in pregnancy are a relatively common occurrence, with a prevalence of $1.4 \%-2 \%$. Most fibroids remain uncomplicated during pregnancy. Degeneration has been estimated to occur in $10 \%$ of fibroids during pregnancy, and is characterized by abdominal pain, leukocytosis, and mild fever [2].

Enterouterine fistulas can occur following: (1) carcinoma of either

Received: Oct 16, 2015 · Revised: Dec 4, 2015 · Accepted: Dec 23, 2015 Corresponding author: Ayman Shehata

Lecturer of Obstetrics and Gynecology, Tanta University, Tanta-El Geish Street 31111, Tanta, Egypt

Tel: +20-12-2588-0071 Fax: +20-40-3320-4569

E-mail:ayman.dawood@med.tanta.edu.eg

This is an Open Access article distributed under the terms of the Creative Commons Attribution Non-Commercial License (http://creativecommons.org/licenses/by-nc/3.0/) which permits unrestricted non-commercial use, distribution, and reproduction in any medium, provided the original work is properly cited. the intestine or uterus with subsequent invasion of the other structure and communication of the lumina, (2) rupture of the uterus (either spontaneous or produced during labor), or (3) inflammatory disease (such as pathological puerperium and appendicular abscess) causing peritonitis, diverticulitis with abscess formation and Crohn disease [3].

\section{Case report}

A 25 -year-old patient presented with an $8 \times 7-\mathrm{cm}$ posterior wall fibroid diagnosed for the first time during pregnancy at 12 weeks. The course of the pregnancy was smooth, with mild enlargement of the fibroid to the size of $10 \times 9 \mathrm{~cm}$.

The patient delivered by Caesarean section at a private clinic due to malpresentation (breech presentation). In the first week postpartum, the patient experienced malodorous vaginal discharge. The patient sought medical advice and antibiotics were given for 14 days, but the discharge did not resolve and the patient felt a mass inside the vagina. The patient presented to Tanta University Hospitals where she was admitted, and under general anesthesia, an examination revealed a solid mass extending from the cervix into the vagina. A biopsy was taken from the mass for histopathological examination, and it was 
identified as a degenerated cellular fibroid polyp.

Antibiotics also were given for 14 days after a culture and sensitivity test, but the discharge still did not resolve. At this point, the patient became frustrated and sought medical advice from more senior practitioners, who advised her to receive gonadotropin-releasing hormone analogues for 2 months. Nonetheless, the discharge was not cured; it became more malodorous as well as greenish and stoollike in character.

The patient developed abdominal pain, distension, vomiting, and absolute constipation 1 week later and was readmitted to Tanta Emergency Hospitals where thorough investigations were performed, including a pelvic ultrasound and a computed tomography scan with gastrointestinal tract contrast.

An examination revealed cachexia and toxicity facies, The patient's vital signs were a blood pressure of $100 / 60 \mathrm{~mm} \mathrm{Hg}$, a pulse of 110 beats per minute and a temperature of $38.1^{\circ} \mathrm{C}$. An abdominal examination revealed distension, tenderness with guard and rigidity, and resonance over all quadrants of the abdomen. No peristalsis was heard over any region of the abdomen.

A pelvic examination demonstrated the presence of tender fornices and a stool-like offensive discharge coming through the vagina, and examination using a speculum found a stool-like discharge coming through the cervix. No fistula was felt through the rectum.

The following laboratory studies were performed: a complete blood count, urinalysis, a liver function panel, a kidney function panel, and serum electrolytes. Ultrasound revealed the absence of peristalsis in the small bowels and a large posterior wall fibroid measuring $10 \times 7 \mathrm{~cm}$ with degeneration. Computed tomography with gastrointestinal tract contrast revealed fistulous communication between the ileum and uterus.

\section{Operative details}

Written consent was obtained from the patient and her relatives for

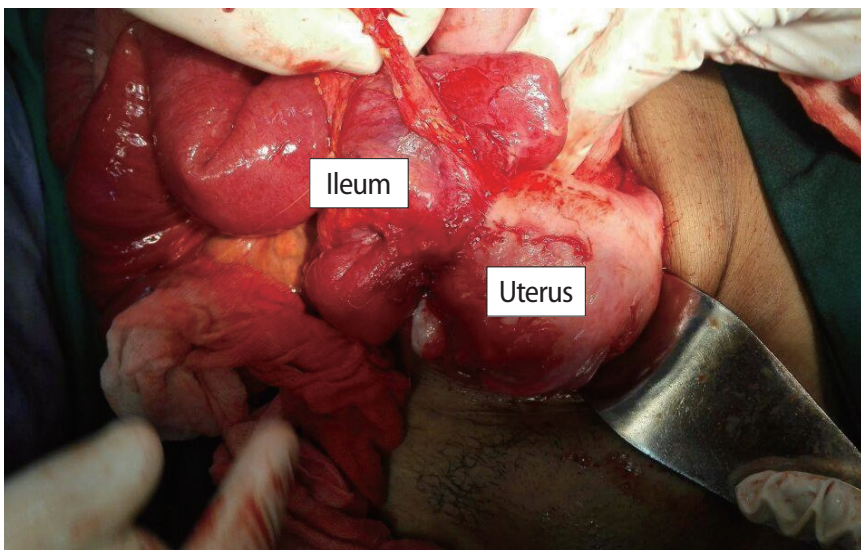

Figure 1. The bowels were adherent to the posterior wall of the uterus. a range of treatment options, including hysterectomy.

Two units of blood were prepared and general anesthesia was administered. A midline infraumbilical incision was made by the surgical team, and we found the abnormal invasion of the fibroid to the ileum approximately $30 \mathrm{~cm}$ from the ileocecal junction (Figure 1).

Dissection of the intestine from the uterus showed the site of the fistula very clearly at the posterior wall of the uterus through the fibroid (Figure 2).

The surgical team managed the case by resection and reanastomosis. The gynecologists managed the case by myomectomy and repair of the endometrial cavity with placement of an intrauterine catheter for antiseptic washing and to prevent Asherman syndrome (Figure 3). Two intraperitoneal drains were inserted, and the catheter was removed 7 days after the operation.

\section{Discussion}

A fistula is defined as an abnormal connection between two epi-

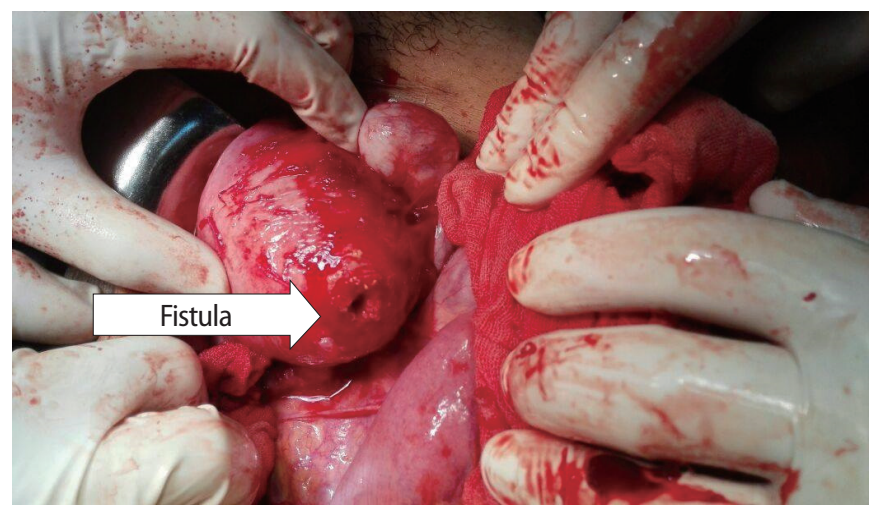

Figure 2. The ileo-uterine fistula observed after separation of the bowel loops.

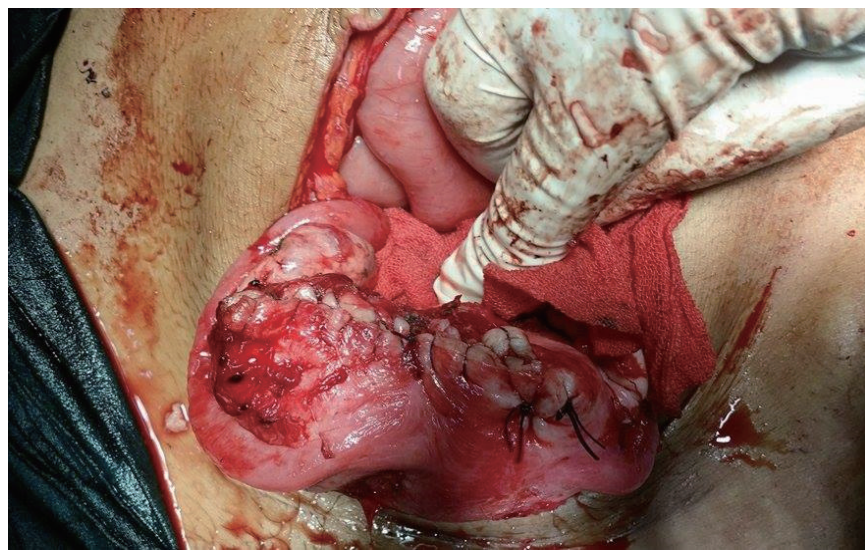

Figure 3. Shape of the uterus after myomectomy and repair of the posterior uterine wall using a three-way Foley catheter. 
thelium-lined organs that do not connect. In the uterus, fistulas can develop to the bladder, colon, and small intestine. Enterouterine fistulas occur infrequently. Martin et al. [4] published perhaps the largest review of enterouterine fistulas in 1956, describing 80 cases, 42 of which followed an obstetric injury, 17 resulted from inflammatory processes, 12 followed curettage, and nine were related to carcinoma. Enterouterine fistulas may be complications of uterine artery embolization as a treatment for myoma $[5,6]$. They may also occur as complications of dilatation and curettage, with a range of post-abortive times of presentation $[7,8]$. They may also be a complication of ectopic pregnancy [9].

An enterovaginal fistula was described by Meier-Boni et al. [10] in 1990. In that case, an enterovaginal fistula was found 1 year after vaginal hysterectomy; radiological imaging revealed a fistula between the terminal ileum and the vagina in an 81-year-old patient. Bacteriological investigations revealed that actinomycosis was the cause of the fistula.

Ileo-uterine fistulas are particularly rare. Fadel [11] described an ileo-uterine fistula after myomectomy that was asymptomatic and discovered accidentally during hysterosalpingography for infertility.

In our case, we found a fistulous tract between a degenerated posterior wall fibroid and the ileum, and administered a questionnaire to patient and her relatives in an attempt to identify the causes of that complication. Several factors may have contributed to the formation of the fistula, with possible explanations including post-Caesarean infection, operative trauma to the bowel during the Caesarean section leading to an infection-caused adhesion between the fibroid and the injured bowel facilitating fistula formation, and potential uterine perforation during uterine sounding - a preliminary step when excising the uterine polyp — which could have led to an infection at that site that may have facilitated fistula formation.

This case was managed surgically by both surgical and gynecological teams. The surgical team excised the fistula and re-anastomosed the gut, following a procedure similar to the operative steps carried out by Meier-Boni et al. [10].

Myomectomy and repair of the uterine cavity was performed by a gynecology team, with insertion of an intra-uterine Foley catheter for seven days to prevent Asherman syndrome.

Our management strategy was in agreement with McFarlane et al. [7] and Duttaroy and Madhok [8], who preserved the uterus, whereas Dewdney et al. [5] and Gutierrez et al. [6] preferred hysterectomy, because the patients in their cases were 50 and 44 years of age, respectively. lleo-uterine fistula is a rare complication of uterine fibroids that occurred in our patient following degenerative changes in a myoma during pregnancy with presentation following delivery by Caesarean section. It was treated by myomectomy and conservative treatment of the uterus after division of the bowel loops and resection and anastomosis of the bowel ends.

\section{Conflict of interest}

No potential conflict of interest relevant to this article was reported.

\section{References}

1. Walker $C L$, Stewart EA. Uterine fibroids: the elephant in the room. Science 2005;308:1589-92.

2. Katz VL, Dotters DJ, Droegemeuller W. Complications of uterine leiomyomas in pregnancy. Obstet Gynecol 1989;73:593-6.

3. Barnes SA, Lillemoe KA. Small intestinal fistulas. In: Zuidema G, Yeo CJ, editors. Shackelford's surgery of the alimentary tract. 5th ed. Philadelphia:W. B. Saunders; 2002. p.29.

4. Martin DH, Hixson CH, Wilson EC Jr. Enterouterine fistula: review. Report of an unusual case. Obstet Gynecol 1956;7:466-9.

5. Dewdney SB, Mani NB, Zuckerman DA, Thaker PH. Uteroenteric fistula after uterine artery embolization. Obstet Gynecol 2011; 118:434-6.

6. Gutierrez LB, Bansal AK, Hovsepian DM. Uteroenteric fistula resulting from fibroid expulsion after uterine fibroid embolization: case report and review of the literature. Cardiovasc Intervent Radiol 2012;35:1231-6.

7. McFarlane ME, Plummer JM, Remy T, Christie L, Laws D, Richards $\mathrm{H}$, et al. Jejunouterine fistula: a case report. Gynecol Surg 2008;5: 173-5.

8. Duttaroy DD, Madhok BM. Jejuno-uterine fistula. Eur J Obstet Gynecol Reprod Biol 2006;129:94-5.

9. Peters IB, Williams CA, Dahniya MH. Uteroenteric fistula due to ruptured ectopic pregnancy. Br J Radiol 1983;56:881-3.

10. Meier-Boni L, Satz N, Hollinger A, Tamborini P. Abdominal actinomycosis: ileo-vaginal fistula as a clinical manifestation. Case report and review of the literature. Wien Klin Wochenschr 1990; 102:21-3.

11. Fadel HE. Ileo-uterine fistula as a complication of myomectomy: case report. Br J Obstet Gynaecol 1977;84:312-3. 